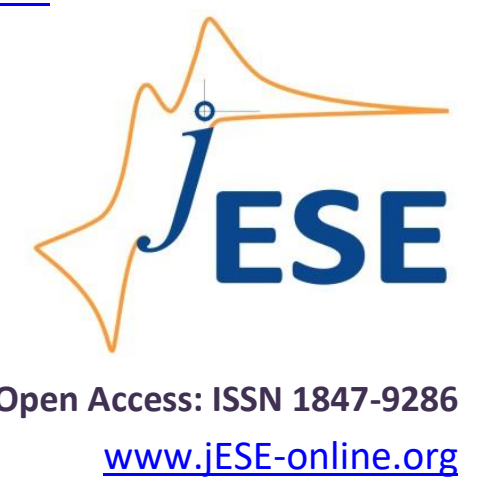

Original scientific paper

\title{
The effects of time-variance on impedance measurements: examples of a corroding electrode and a battery cell
}

\author{
Nicolas Murer ${ }^{\bowtie}$, Jean-Paul Diard and Bogdan Petrescu \\ Bio-Logic SAS, 4 Rue de Vaucanson, 38170 Seyssinet-Pariset, France \\ Corresponding author: ${ }^{\bowtie i c o l a s . m u r e r @ b i o-l o g i c . n e t, ~ T e l .: ~+334 ~} 76986831$ \\ Received: September 17, 2019; Accepted: October 10, 2019
}

\begin{abstract}
When performing electrochemical impedance spectroscopy (EIS) measurements on a system, we must make sure it fulfills certain conditions. One of them is that it should be stationary that is to say, steady-state and time-invariant. Commonly studied systems are time-variant, for example a corroding electrode or a battery under operation. A corroding electrode sees its polarization resistance decrease with time. A passivating electrode sees its polarization resistance increase with time. These phenomena cause a deformation of the Nyquist impedance at low frequencies. This result was first simulated and validated by experimental measurements on a corroding steel sample undergoing uniform corrosion. The effect of performing impedance measurements on a discharging battery was also shown. Several methods are available to check and correct time-variance. The nonstationary distortion (NSD) indicator is used to separate valid and invalid data samples and the so called " $4 D$ impedance" method can easily produce instantaneous impedance data.
\end{abstract}

\section{Keywords}

EIS; non-stationarity; corrosion; in operando; battery cells; 4D impedance; NSD.

\section{Introduction}

For a valid impedance measurement, the system under investigation should be linear, stable, causal and stationary [1,2]. These properties, which imply stationarity can be tested and checked using Kramers-Kronig [1] and Hilbert transforms [3].

In this paper, the term "stationarity" comprises steady-state and time-invariance.

Steady-state is the state of a system after its transient state. For example, an R/C circuit with a definite time-constant is submitted to a potential or current step. Its response will vary over time after reaching a steady-state. Another example is any system submitted to a series of AC potential or current modulations of different frequencies, which is basically what an electrochemical 
impedance spectroscopy (EIS) measurement is. When the frequency is being switched from one value to another, the system response is transient.

Time-variance is the property of a system whose parameters defining its transfer function change with time. For example, a corroding electrode whose polarization resistance changes over time, either because of corrosion or of passivation, is a time-variant system. A discharging battery also sees its various resistances (charge transfer, diffusion) evolve during discharge. Sometimes steadystate and time-invariance are difficult to distinguish.

The most classical use of EIS in corrosion is for the determination of the polarization resistance $R_{\mathrm{p}}$ using the Wagner-Traud relationship [4-8]. A corroding system is specifically a non-stationary system after the first instant of immersion. The change in parameters can greatly affect the impedance data especially at lower frequencies.

In the field of batteries, EIS measurements during continuous charge or discharge, or in operando, are performed to investigate how a battery alters while it is being operated [9-14]. The problem in this case is to know which impedance data can be considered valid, or more importantly, which frequency data should be considered erroneous because of the time-variance of the system.

This problem of non-stationarity has been known for quite some time now [11] and various methods have been proposed to check and correct the effect of non-stationarity on impedance measurements.

In this paper, first we tried to simulate the effect of a polarization resistance that changes with time on the impedance data of a simple corroding system whose DC characteristic follows the Wagner-Traud relationship. The deformation of the impedance diagrams at low frequencies were confirmed by experimental data obtained in acidic media on a stainless steel sample. Secondly, impedance data were measured on a discharging battery, to show the effect of time-variance. Finally, several methods are presented demonstrating how to check and correct the effect of timevariance: i) using galvano-controlled EIS instead of potentio-controlled EIS, ii) performing two successive EIS measurements, iii) using a non-stationarity distortion indicator to separate valid and invalid impedance data and iv) using the 4D impedance representation to easily plot instantaneous impedance diagrams.

\section{Simulation conditions}

Let us consider a simple corroding system for which the steady-state behavior is described by the Wagner-Traud relationship, derived from the Butler-Erdey-Grúz-Volmer relationship, better known as the Butler-Volmer relationship:

$$
I=I_{\text {corr }}\left(\exp \left(b_{1}\left(E-E_{\text {corr }}\right)-\exp \left(-b_{2}\left(E-E_{\text {corr }}\right)\right)\right)\right.
$$

where $I$ is the current in $A, I_{\text {corr }}$ the corrosion current in $A, E$ the potential in $V, E_{\text {corr }}$ the corrosion potential in $\mathrm{V}, b_{1}$ and $b_{2}$ the Tafel slopes in $\mathrm{V}^{-1}$.

Assuming this relationship holds when the system is submitted to a dynamic perturbation and that this perturbation $\Pi=E-E_{\text {corr }}$ is small enough, the Faradaic impedance of the considered system is the charge transfer resistance $R_{c t}$ expressed as:

$$
R_{\mathrm{ct}}=Z_{\mathrm{f}}=\frac{1}{I_{\text {corr }}\left(b_{1} \exp \left(b_{1} \Pi\right)+b_{2} \exp \left(-b_{2} \Pi\right)\right)}
$$

More details on this calculation can be found in $[15,17]$. Adding the electrolyte resistance $R_{\Omega}$ in series and the double layer capacitance $C_{d l}$ in parallel, the simplest way to model a corroding 
electrode is to use the equivalent electrical circuit shown in Fig. 1 with its corresponding impedance Nyquist diagram (Fig. 1).
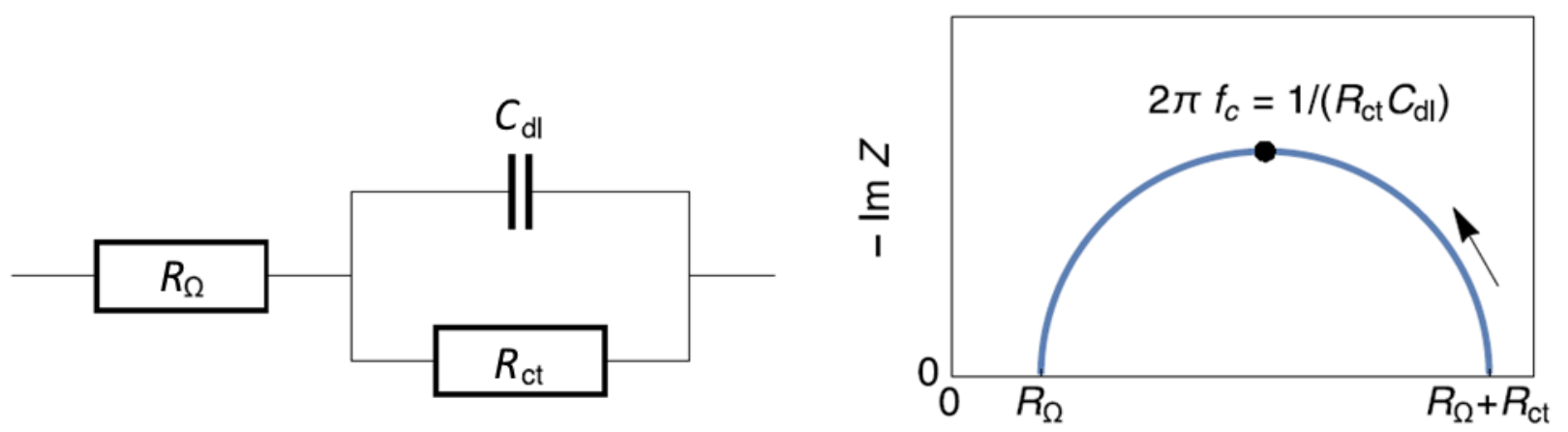

$\operatorname{Re} Z$

Figure 1. Left - Equivalent circuit corresponding to an electrode in solution whose DC behavior follows the Wagner-Traud expression; Right - Corresponding Nyquist representation of its impedance.

In the above example $R_{\mathrm{ct}}=R_{\mathrm{p}}$. The analytical expression of the impedance $Z$ of a Tafelian system in solution is known and can be simulated using Mathematica [17].

$$
z(j \omega)=R_{\Omega}+\frac{R_{\mathrm{p}}}{1+j \omega C_{\mathrm{dl}} R_{\mathrm{p}}}
$$

where $j=\sqrt{ }-1, \omega$ the modulation pulse in $\mathrm{rad} \mathrm{s}^{-1}, R_{\Omega}$ the electrolyte resistance in $\Omega$ and $C_{\mathrm{dl}}$ the double layer capacitance at the electrode interface in $\mathrm{F}$.

Two cases were simulated, first a polarization resistance that is decreasing with time and second a polarization resistance that is increasing with time following these relations:

$$
\begin{aligned}
& R_{\mathrm{p}}(t)=R_{\mathrm{p}}(0)-k \sqrt{ } t \\
& R_{\mathrm{p}}(t)=R_{\mathrm{p}}(0)-k t^{2}
\end{aligned}
$$

Where $R_{\mathrm{p}}(0)$ is the value of the polarization resistance at the beginning of the simulation $(t=0)$ in $\Omega$, and $k$ a constant factor in $\Omega \mathrm{s}^{-1 / 2}$ for Eq. (4) and in $\Omega \mathrm{s}^{-2}$ for Eq. (5).

\section{Experimental conditions}

\section{EIS measurement on a corroding sample}

Potentio-controlled EIS measurements were performed on a large mild steel sample (undisclosed composition) using a coating cell, a carbon rod electrode, an $\mathrm{Ag} / \mathrm{AgCl}$ reference electrode and $0.1 \mathrm{M}$ $\mathrm{H}_{2} \mathrm{SO}_{4}$ solution.

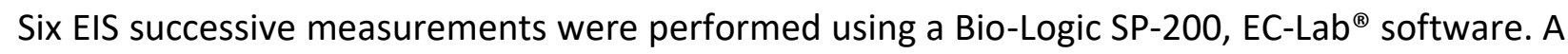
$20 \mathrm{mV}$ voltage amplitude modulation was applied around OCV with frequencies ranging from $200 \mathrm{kHz}$ to $10 \mathrm{mHz}$ with 6 points per frequency decade. For each frequency, the result was an average of two measurements.

\section{EIS measurements on discharging battery cell}

A 26650 LFP battery cell produced by $A 123$ was used. A discharge of $-100 \mathrm{~mA}$ during $130 \mathrm{~s}$ was performed followed by a galvano-controlled EIS (GEIS) measurement with a DC current of $-100 \mathrm{~mA}$ and an $A C$ current modulation of $-200 \mathrm{~mA}$ at frequencies ranging from $1 \mathrm{kHz}$ to $10 \mathrm{mHz}$ with 6 points per frequency decade. Using a loop technique, the experiment, composed of a discharge followed by a GEIS, was performed nine times. 


\section{Results}

\section{Simulation}

The following values were used: $R_{\Omega}=50 \Omega, R_{\mathrm{p}}(0)=500 \Omega, k=1.5 \Omega \mathrm{s}^{-1 / 2}$ (decreasing $R_{\mathrm{p}}(t)$ ) or $0.510^{-5} \Omega \mathrm{s}^{-2}$ (increasing $R_{\mathrm{p}}(t)$ ), $C_{\mathrm{dl}}=2 \times 10^{-2} \mathrm{~F}, f_{\max }=10 \mathrm{~Hz}$ and $f_{\min }=10^{-3} \mathrm{~Hz}$. All frequencies are logarithmically distributed over 8 points per decade.

Impedance data are calculated over frequency values $f_{\mathrm{i}}$, which are used to calculate $t$ :

$$
t\left(f_{\mathrm{n}}\right)=\sum_{i=1}^{\mathrm{n}} t\left(f_{\mathrm{i}}\right)=\sum_{\mathrm{i}=1}^{\mathrm{n}} \frac{1}{f_{\mathrm{i}}}
$$

with $t$ the time in $\mathrm{s}$ and $f$ the frequency in $\mathrm{Hz}$. Please note that this formula assumes that for each frequency, only one period is used for the simulation, which is not the case in a real measurement, where at high frequencies, generally several periods of the signal are used to measure the impedance. The obtained results are shown in Figure 2.
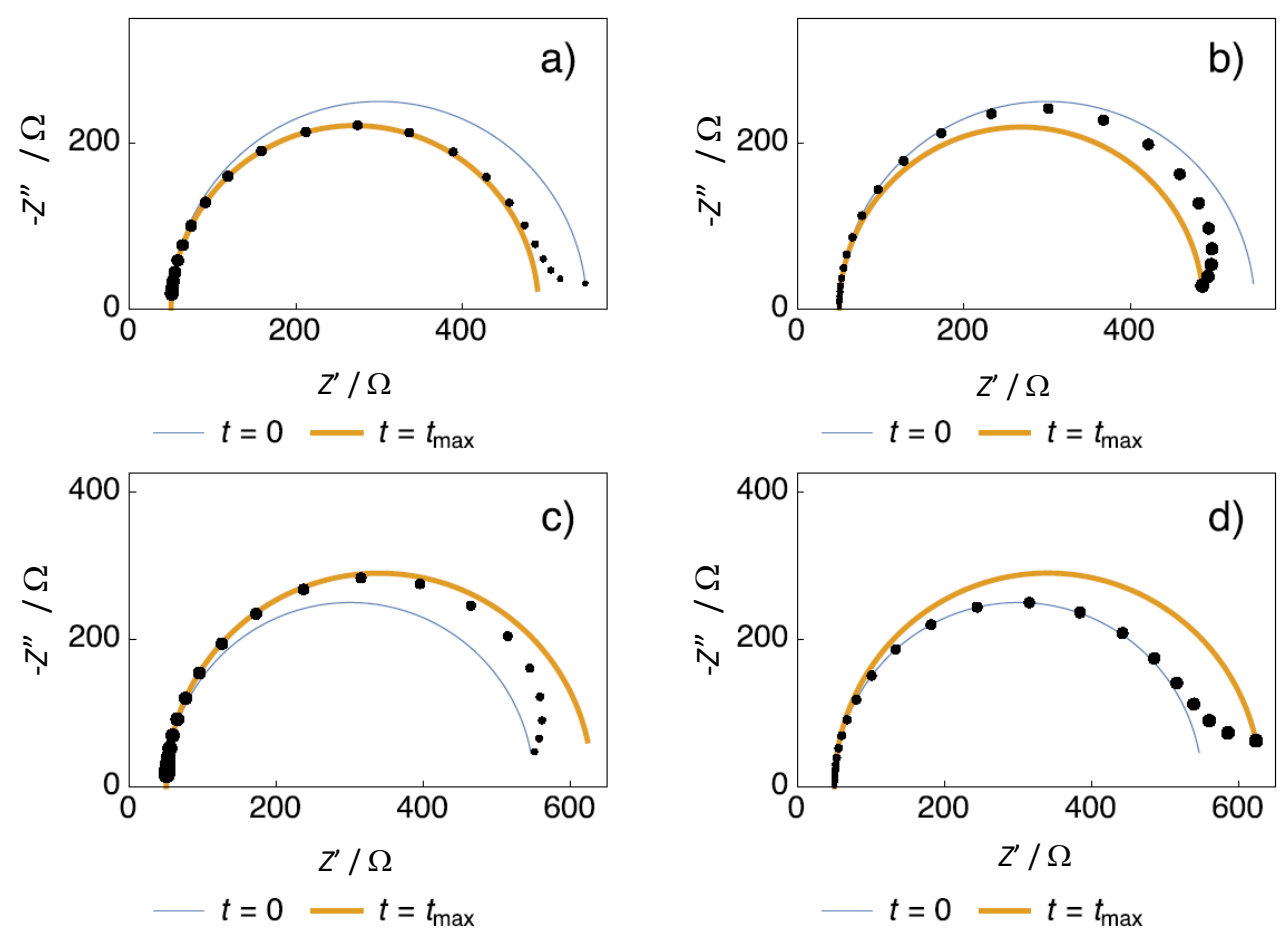

e)

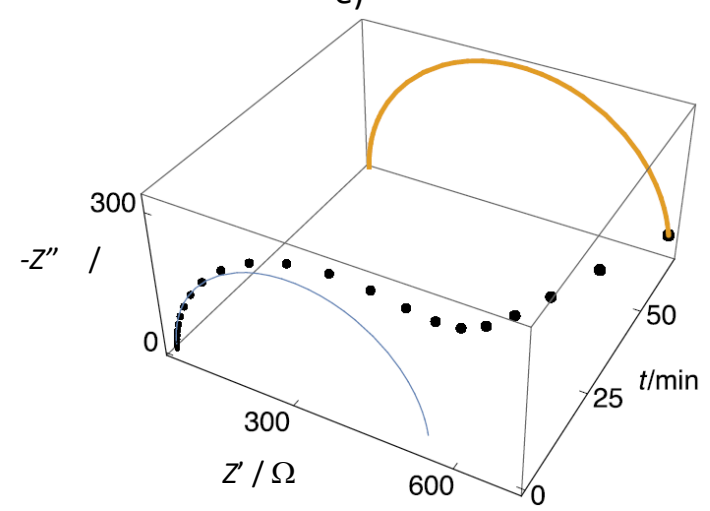

Figure 2. Nyquist representation of impedance data obtained using Eq. (3) and parameters written above with dots: $\mathrm{R}_{p}(\mathrm{t})$; thin blue line: $R_{p}(0)$; thick orange line: $\mathrm{R}_{p}\left(\mathrm{t}=\mathrm{t}_{\max }\right)(a)$ for $a$ decreasing $R_{p}(t)$ scanning from $\mathrm{f}_{\min }$ to $\mathrm{f}_{\max }(\boldsymbol{b})$ for a decreasing $\mathrm{R}_{p}(\mathrm{t})$ scanning from $\mathrm{f}_{\max }$ to $\mathrm{f}_{\min }(\boldsymbol{c})$ for an increasing $\mathrm{R}_{p}(\mathrm{t})$ scanning from $\mathrm{f}_{\min }$ to $\mathrm{f}_{\max }(\mathrm{d})$ for an increasing $\mathrm{R}_{p}(\mathrm{t})$ scanning from $\mathrm{f}_{\max }$ to $f_{\min }(e) 3 D$ representation of the results shown in $(d)$. The size of the dots increases with time 
It can be seen in Figure 2 that as time goes by, the data points move slowly from the thin blue curve to the thick orange curve, which is to be expected, as the thin blue curve is the instantaneous impedance diagram at $t=0$ and the thick orange curve is the instantaneous impedance diagram at $t=t_{\text {max }}$. With a decreasing $R_{\mathrm{p}}(t)$ and scanning from high to low frequencies the Nyquist graph is "folding over" smaller Re $Z$ values at lower frequencies (Fig. 2a). A similar shape can be seen with an increasing $R_{\mathrm{p}}(\mathrm{t})$ when scanning from low to high frequencies (Fig. 2c). With a decreasing $R_{\mathrm{p}}(t)$ and scanning from low to high frequencies, the Nyquist graph forms a tail over larger $\operatorname{Re} Z$ values, at lower frequencies ie longer times (Fig. 2b). The same shape can be seen with an increasing $R_{\mathrm{p}}(\mathrm{t})$ and scanning from high to low frequencies (Fig. $2 d$ ). Figure $2 \mathrm{e}$ is a pseudo 3D representation of Figure $2 \mathrm{~d}$.

\section{Corrosion experiment}

The results are shown in Figure 3. Please note that the frequency sweep was performed from high to low values, corresponding in the simulation data to Figures $2 b$ and $2 d$. It can be seen in Figure 3a that right after immersion (Graph 1 in Fig. 3a), the instantaneous polarization resistance seems to increase and then to rapidly decrease, hence the occurrence of a loop at lower frequencies (Fig. 3b).

The next five impedance diagrams show the "fold-over" of the values at lower frequencies, which, according to Figure $2 a$ is characteristic of a decrease of the instantaneous polarization resistance. It should be noted that unlike the simulated data, the measured data show an inductive behavior at lower frequencies, with values that have a positive imaginary part.

We can assume, considering the $\mathrm{pH}$ of the electrolyte, that a Volmer-Heyrovský mechanism is taking place: it is a common two-step mechanism for hydrogen evolution, with an adsorption step and dihydrogen release step. It was calculated that the impedance of such a reaction shows an inductive loop at lower frequencies [18].

However, the general trends predicted by the simulation (Fig. 2) seem to be validated by experimental data (Fig. 3a).

a)

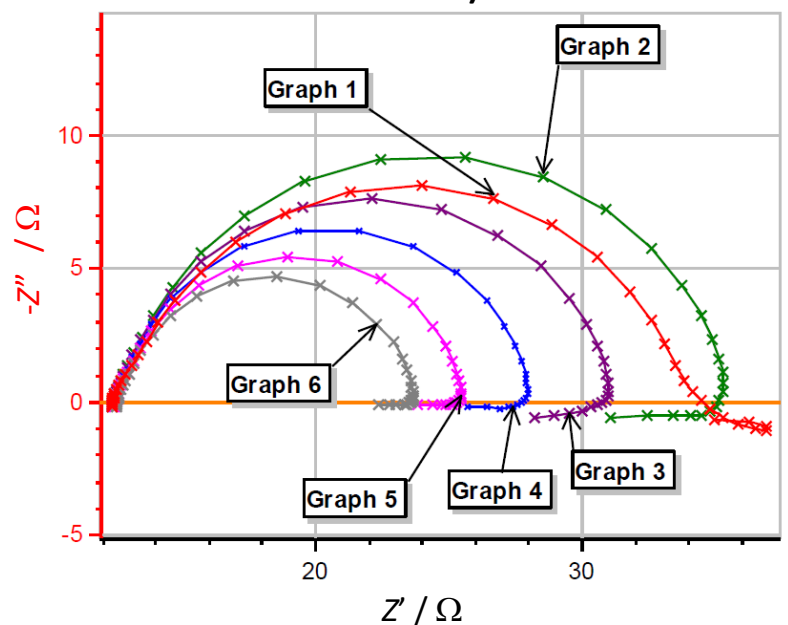

b)

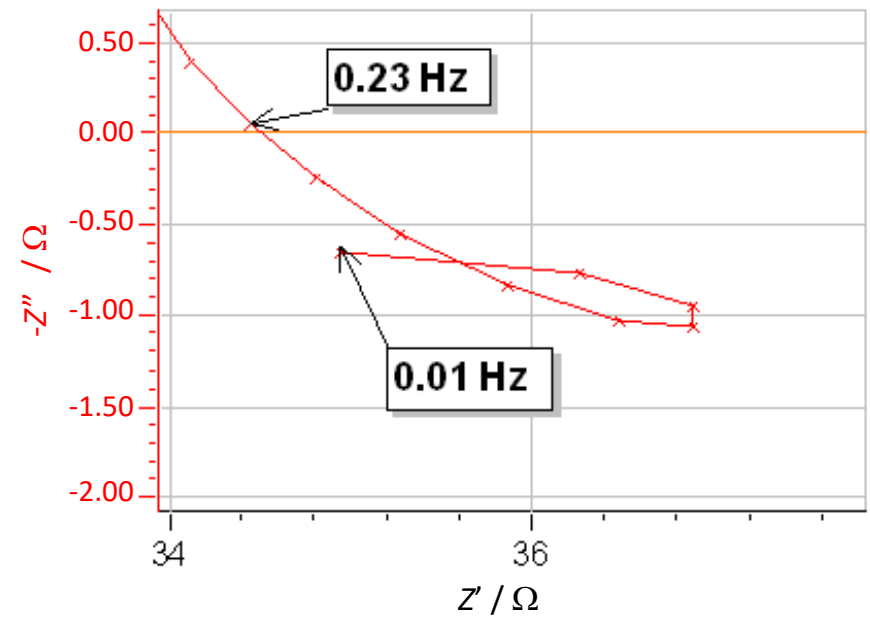

Figure 3. (a) Nyquist representation of the impedance data ( $200 \mathrm{kHz}-10 \mathrm{mHz}$ frequency range) obtained on a mild steel sample (undisclosed composition) just after immersion in $0.1 \mathrm{M} \mathrm{H}_{2} \mathrm{SO}_{4}$. The six measurements were performed one after another. (b) Close-up on the low frequency part of the first obtained graph

\section{Battery experiment}

Figure 4 shows the successive impedance diagrams obtained on the batteries under discharge. Before each impedance measurement, the battery is being discharged during $130 \mathrm{~s}$ at $-100 \mathrm{~mA}$. The 
impedance measurement is performed with galvanic control hence there is no discontinuity between the discharge and the impedance measurement. Figures $4 \mathrm{a}$ to $4 \mathrm{e}$ show the first five successive measurements. Figure $4 \mathrm{f}$ is the last measured impedance diagram, i.e. the tenth impedance diagram.

a)

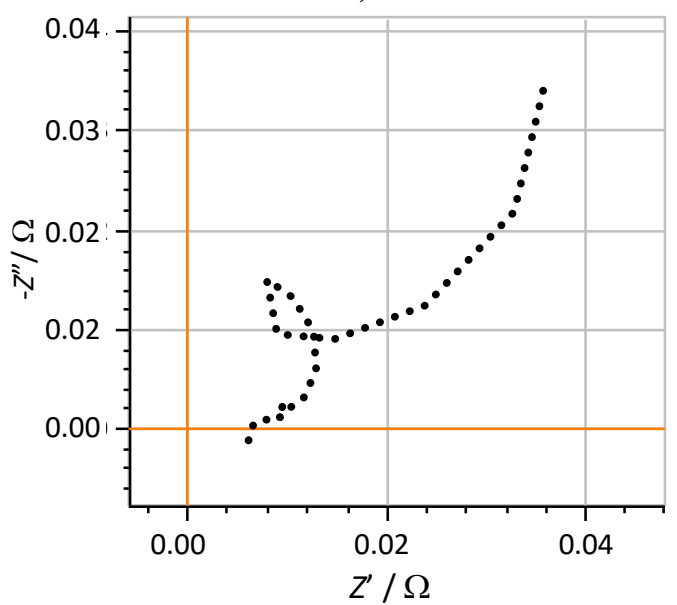

c)

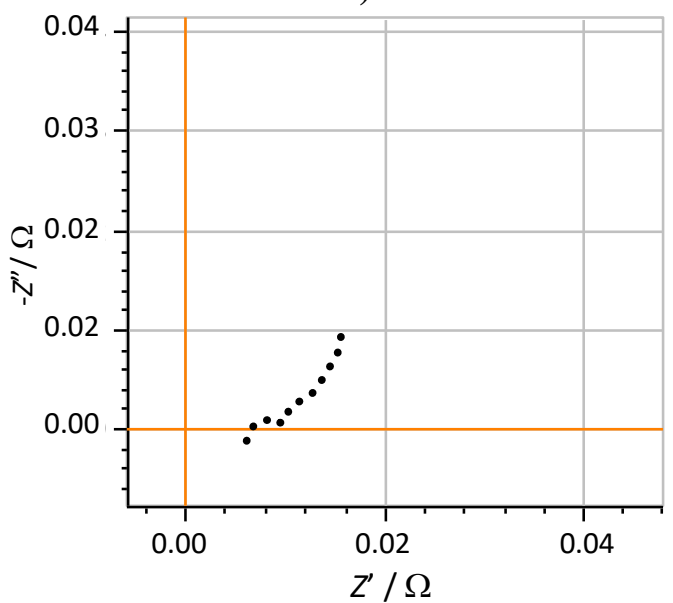

e)

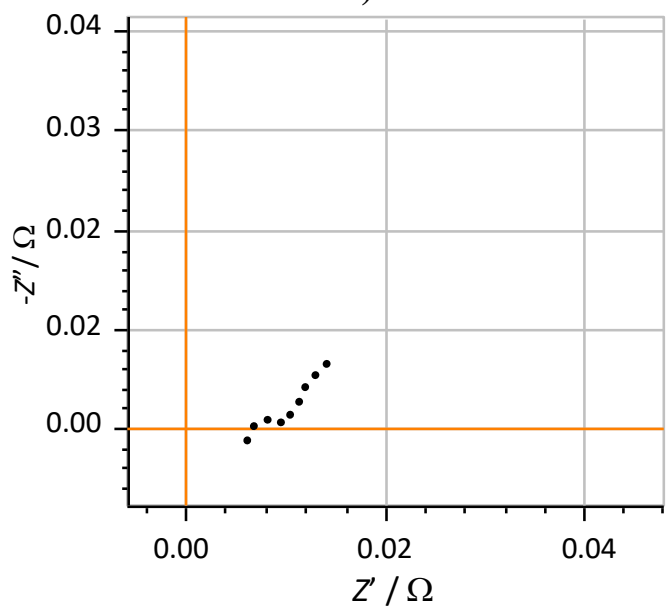

b)

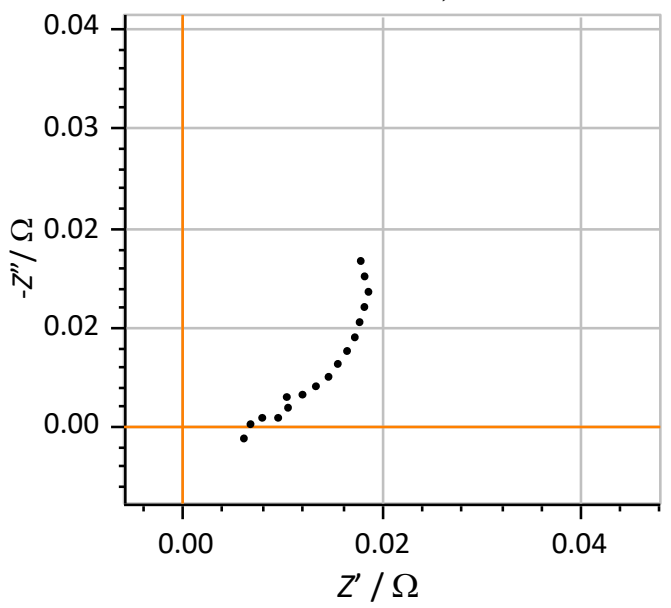

d)

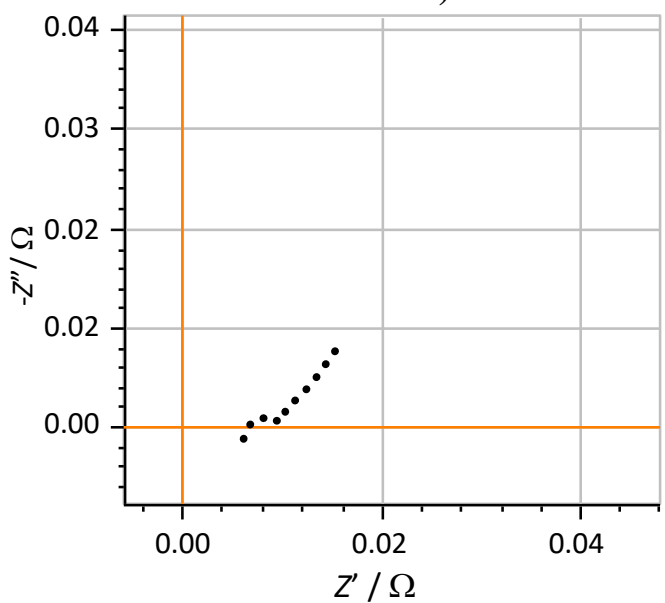

f)

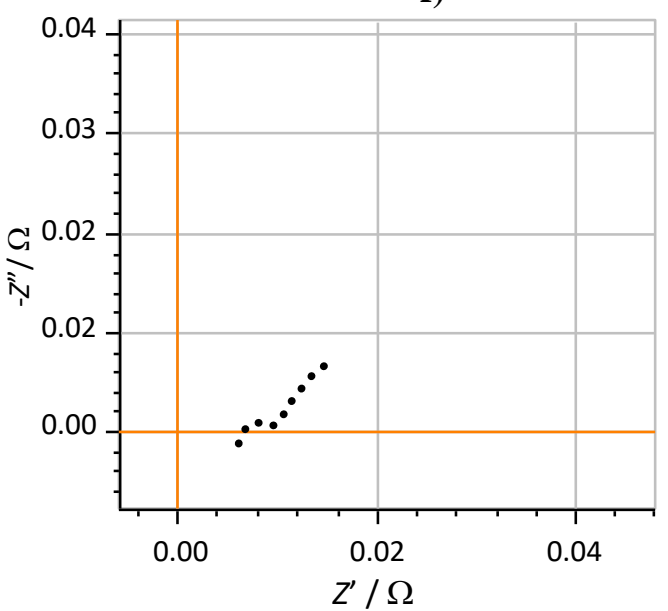

Figure 4. Nyquist representation of the impedance data obtained on a LFP 26650 battery from A123 using the conditions shown in Fig. 4 (1 kHz-10 mHz frequency range) (a)-(e) Cycles 1-5 (f) Cycle 10. Each impedance measurement was preceded by a discharge at $-100 \mathrm{~mA}$ during $130 \mathrm{~s}$. The DC potential evolution during the discharge and the GEIS experiment are shown in Fig. 5 
The first three diagrams are quite different and then the data seem to stabilize. Figure 5 shows the potential evolution during the discharge and the impedance measurement for cycles 1 to 5 (Fig. 5a to 5e) and cycle 10 (Fig. 5f). We can see that the potential mostly decreases during the first three cycles and then starts to stabilize.

a)

$\times$ GCPL mpr: $E_{\text {we }}$ vs. time, loop 0

- GEIS mpr: $E_{\text {we }} v s$. time, cycle $1 \#$

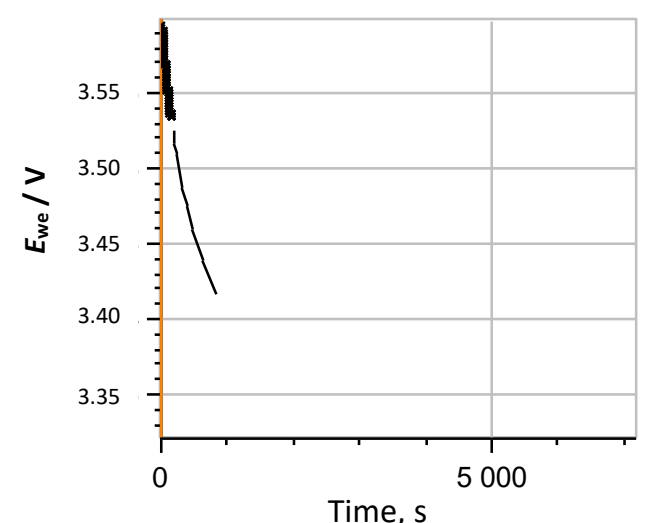

c)

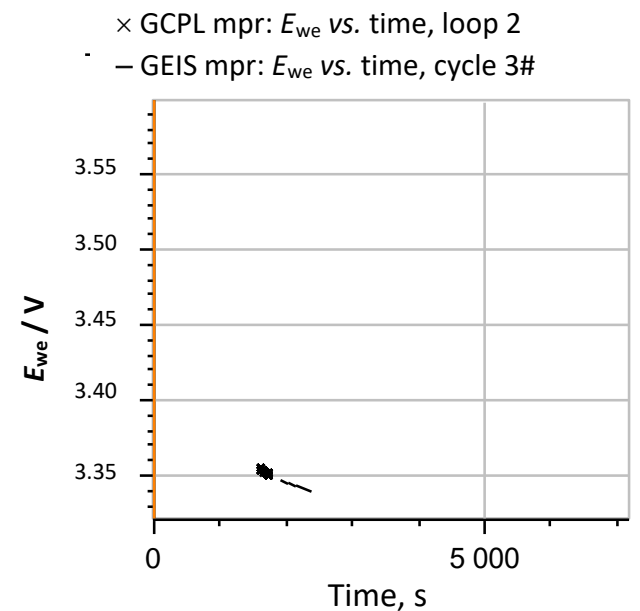

e)

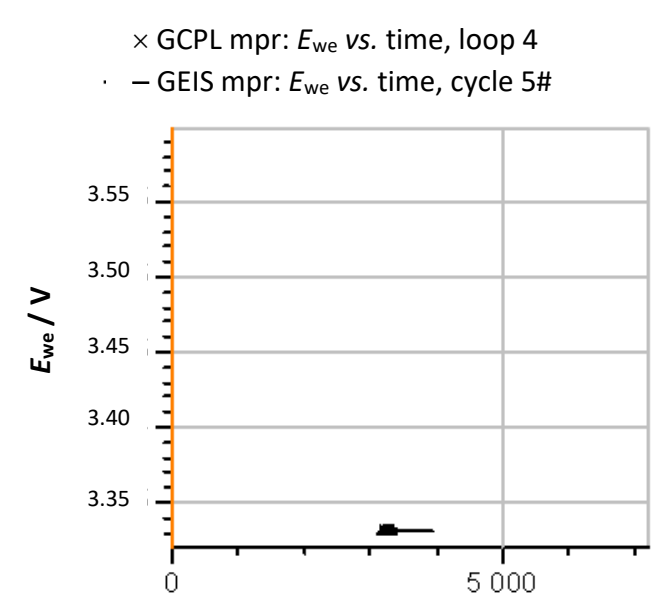

Time, $\mathrm{s}$ b)

$\times$ GCPL mpr: $E_{\text {we }}$ vs. time, loop 1

- GEIS mpr: $E_{\text {we }} v s$. time, cycle $2 \#$

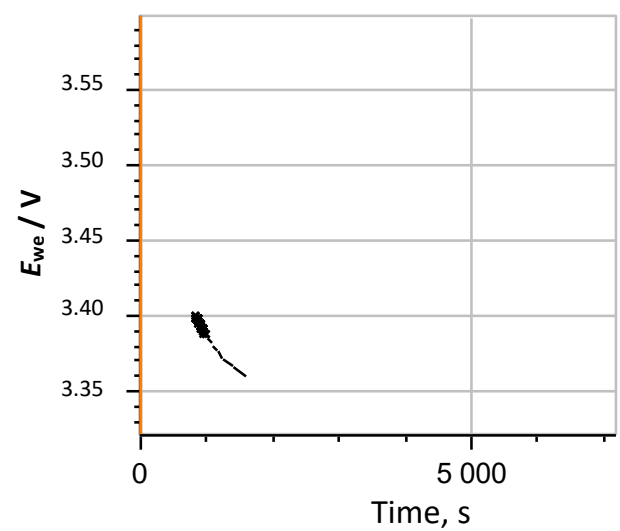

d)

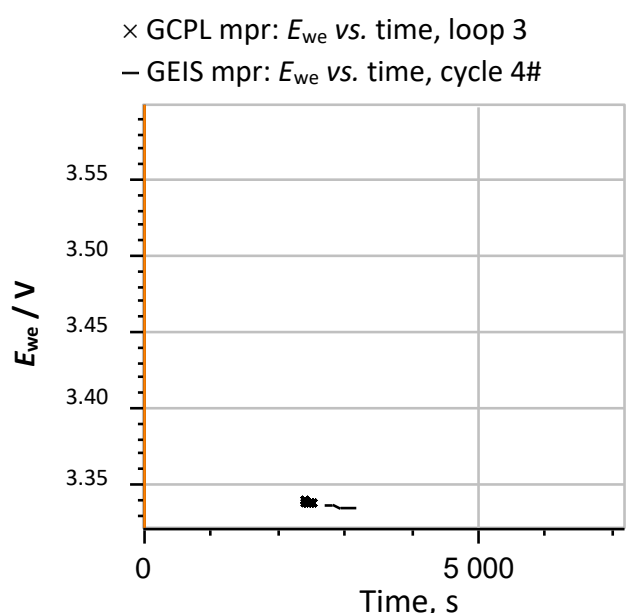

Time,

f)

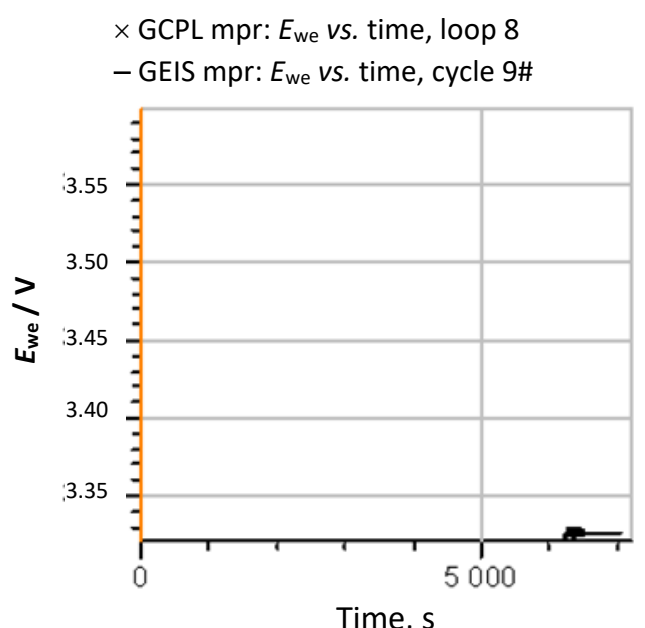

Figure 5. DC Potential evolution obtained on a LFP 26650 battery from A123 during EIS measurements preceded by a discharge at -100 mA during 130 s (a)-(e) Cycles 1-5 (f) Cycle 9.

The DC potential evolution during the discharge is shown by thick markers and during the impedance measurement by a thin line 
The comparison of Figures 4 and 5 shows that the unusual impedance values at low frequencies are due to the time-variance of the system, which is directly related to the DC current applied to the battery. It is quite difficult to interpret these data, and the peculiar shape of the impedance data at lower frequencies is not related to a specific electrochemical phenomenon but to the change of the systems parameters and a transient behavior.

Which data can be reliably interpreted and considered as valid? How can I make sure that my interpretation of the data is not erroneous, for example that the time-variance effect is not interpreted as an inductive loop? In the next part of this paper, we will present some tools that will help address such problems.

\section{Discussion: How to check and correct time-variance?}

\section{Perform successive EIS measurements}

An obvious and easy means of checking time-variance is to perform successive EIS measurements, scanning frequencies either in the same direction each time or reversing the order, that is to say from high to low and back to high frequencies. Figure $3 a$ is a good example of such a measurement.

\section{Perform EIS with galvano control}

Impedance spectroscopy can be performed by controlling the potential, which means that the input signal is a potential modulation around a DC potential and the response of the system is a current modulation around a DC current. In this case, the transfer function is not the impedance but the admittance of the system. However, the input modulation can also be an AC current modulation around a DC current, in which case the response is an AC potential modulation around a DC potential. In this case, the transfer function is the impedance of the system. The impedance diagrams shown in Fig. 3 were obtained using the potential-controlled EIS (PEIS). Figure 6 shows the evolution of the DC current during this experiment, for which the potential modulation is applied around the Open Circuit Potential (OCP) measured just before the beginning of the measurement.

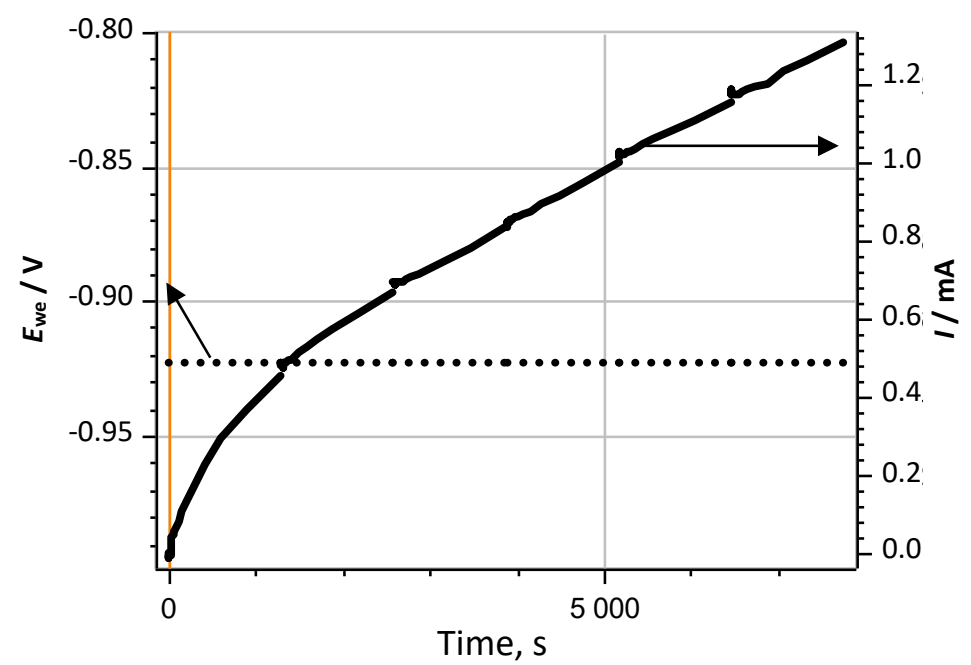

Figure 6. Evolution of DC current and DC potential during the six potentio-controlled EIS experiments shown in Fig. 3.

It can be seen in Figure 6, that the potential is maintained at a constant level over the whole experiment duration but that the DC current is rapidly moving away from zero, meaning that as the time goes by, the sample is anodically polarized due to the evolution of its free corrosion potential 
(or OCP). This phenomenon is illustrated in Figure 7a, which shows that if the steady-state I vs. $E$ characteristic of a corroding sample is moving towards more cathodic potentials, the initial OCP becomes an anodic potential. Potentio-Controlled (PC) impedance measurements are not performed around the initial OCP but around a specific operating point on the anodic part of the steady-state curve. On the contrary, as illustrated in Figure $7 \mathrm{~b}$, if the impedance measurement is performed using current control (or Galvano-Control GC) and around zero current (which is equivalent to $\mathrm{OCP}$ ), even though the steady-state curve is changing and moving towards cathodic potentials, the modulation is still performed around the same point on the steady-state curve.
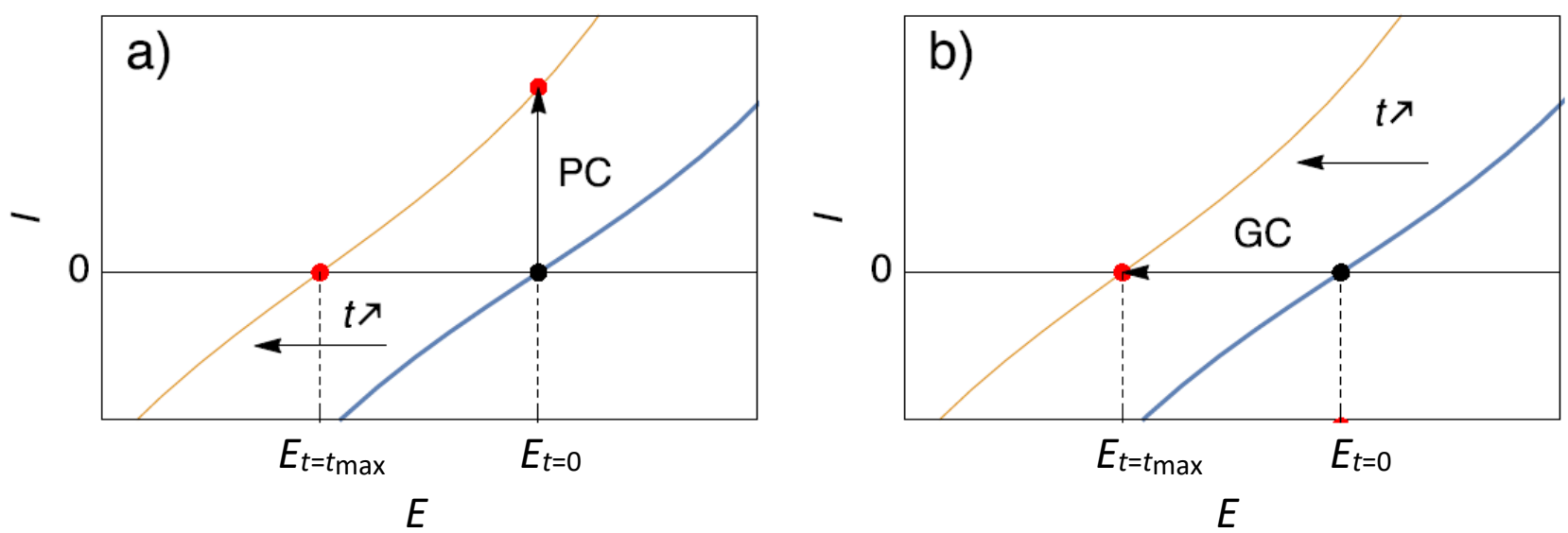

Figure 7. Description of the effect of (a) Potential control (PC) and (b) Galvano control (GC) when the steady-state curve of the system is moving towards more cathodic potentials. Only galvano control at zero current allows to follow the change of the corrosion potential.

The effect of using a controlled current instead of a controlled potential can be seen in Figure 8 . Figure 8a shows the impedance diagram measured while the corrosion potential and the corrosion current change as shown in Figure 7a: the blue $I$ vs. $E$ characteristic is at $t=0$ and the orange $I v s$. $E$ characteristic is at $t=t_{\text {max }}$. Figure $8 \mathrm{~b}$ shows the same impedance diagram but measured using a controlled current. In both cases, the effect of the system's time-variance can be seen at lower frequencies, but this is reduced in the case of a controlled current and is only due to the corrosion current change and not to the corrosion potential change. The fact that the sample is not anodically polarized reduces the extent of the time-variance of the system.
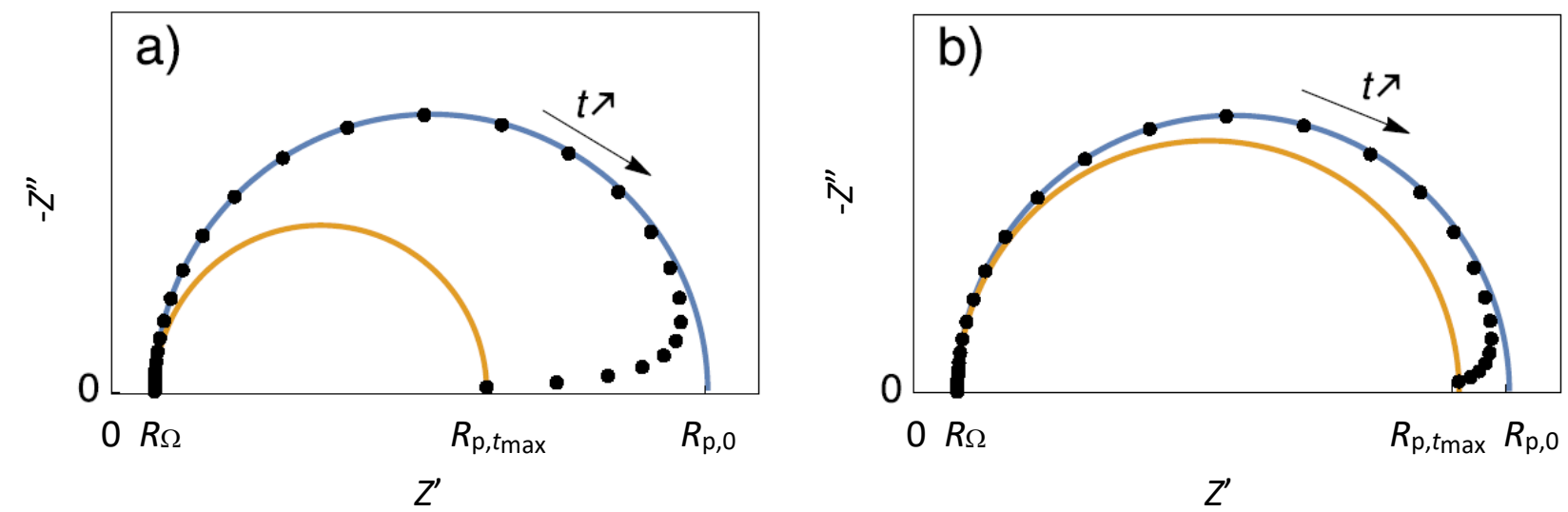

Figure 8. Nyquist representation of simulated impedance data obtained with (a) Potential Control (PC) and

(b) Galvano Control (GC) when the steady-state curve of the system is moving towards more cathodic potentials. In galvano control the time-variance is only due to the change of corrosion current but not to the change of corrosion potential. The parameter values are: $R_{\Omega}=20 \Omega ; C_{\mathrm{dl}}=3 \times 10-3 \mathrm{~F} . R_{\mathrm{p}}(t)$ is given by the $E q$. (2) with $I_{\text {corr }}(t)$ and $E_{\text {corr }}(t)$ changing linearly with time. 


\section{Non-Stationary Distortion (NSD) indicator}

In this paper, as explained in the introduction, what we mean by non-stationarity is two-fold:

i) The fact that the system is in a transient regime that it has not yet reached its steadystate. Its transfer function stays the same throughout the experiment but its steady-state response is not reached instantaneously, it is lagging.

ii) The fact that the system sees its transfer function or the values of the parameters constituting its transfer function change over time. This is what we called throughout the paper time-variance.

Both phenomena have a specific effect on the response signal, which can be seen on the impedance diagram, but also on its Fourier Transform (FT), which gives a frequency representation of the signal. The FT of the response signal of a stationary system will show a line at the same frequency as the input signal (Fig. 9). The response of a non-stationary system, whether it is in a transient state (Fig. 10a) or time-variant (Fig. 10b), will show lines not only at the same frequency as the input signal but also at adjacent frequencies.
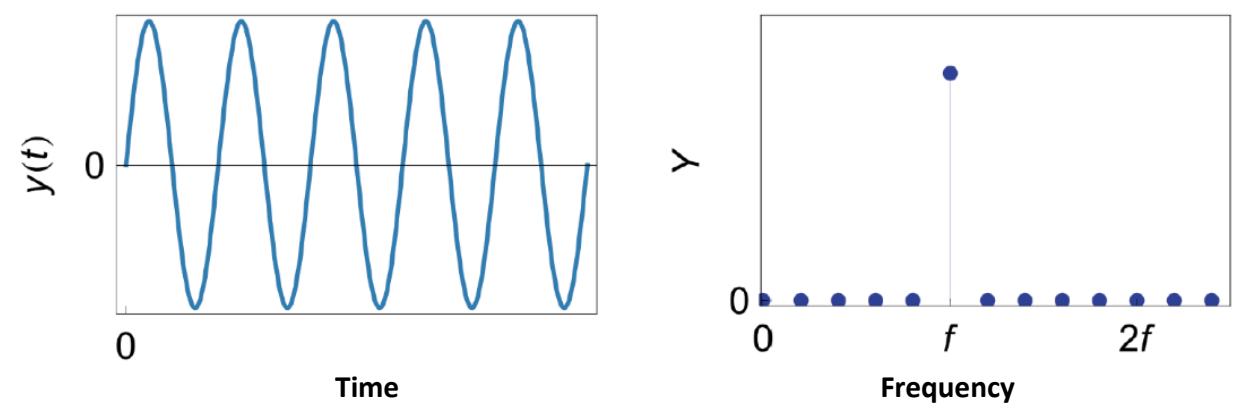

Figure 9. Time and frequency representation of the response of a stationary system to a single sinus wave with a frequency $f$ (taken from [19])

a)
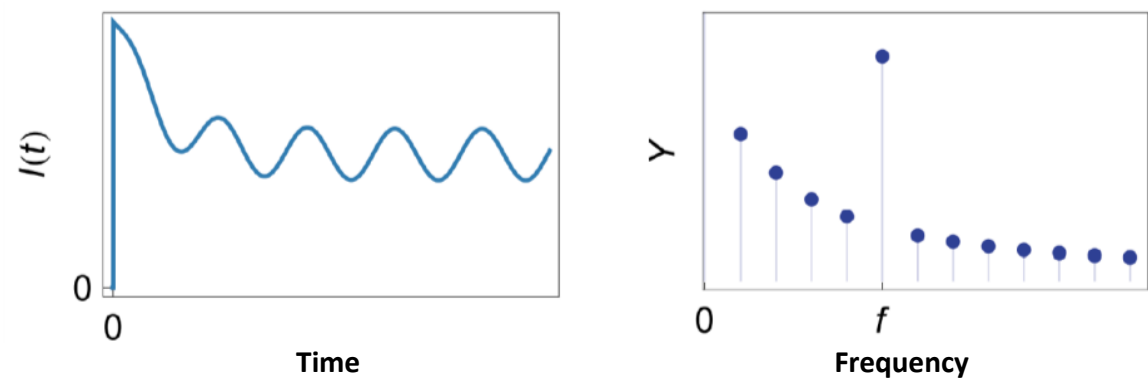

b)
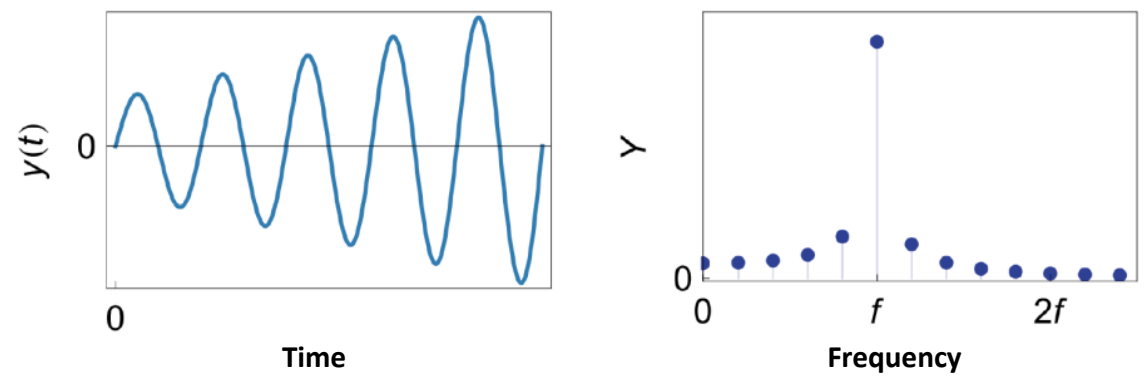

Figure 10. Time and frequency representation of the response of (a) a non-steady state system and (b) a time-variant system to a single sinus wave with a frequency $f$ (taken from [19]).

The amplitude of the adjacent lines around the signal response at the fundamental frequency is proportional to the extent of non-stationarity of the system. 
We can introduce an indicator that can be used to quantify the non-stationarity of the signal, whether it is due to a transient state or to a time-variance. We call this indicator NSD which is calculated as follows:

$$
\operatorname{NSD}(f)=\frac{1}{Y_{f}} \sqrt{Y_{f-\Delta f}^{2}+Y_{f+\Delta f}^{2}}
$$

where $Y_{f}$ is the amplitude at the stimulation or fundamental frequency, $Y_{f-\Delta f}$ and $Y_{f+\Delta f}$ are the amplitudes at the adjacent frequencies to the fundamental and $\Delta f$ is the spectral frequency resolution. Figure 11a shows the NSD for the impedance measurements shown in Figure 4 and again here in Figure 11b.
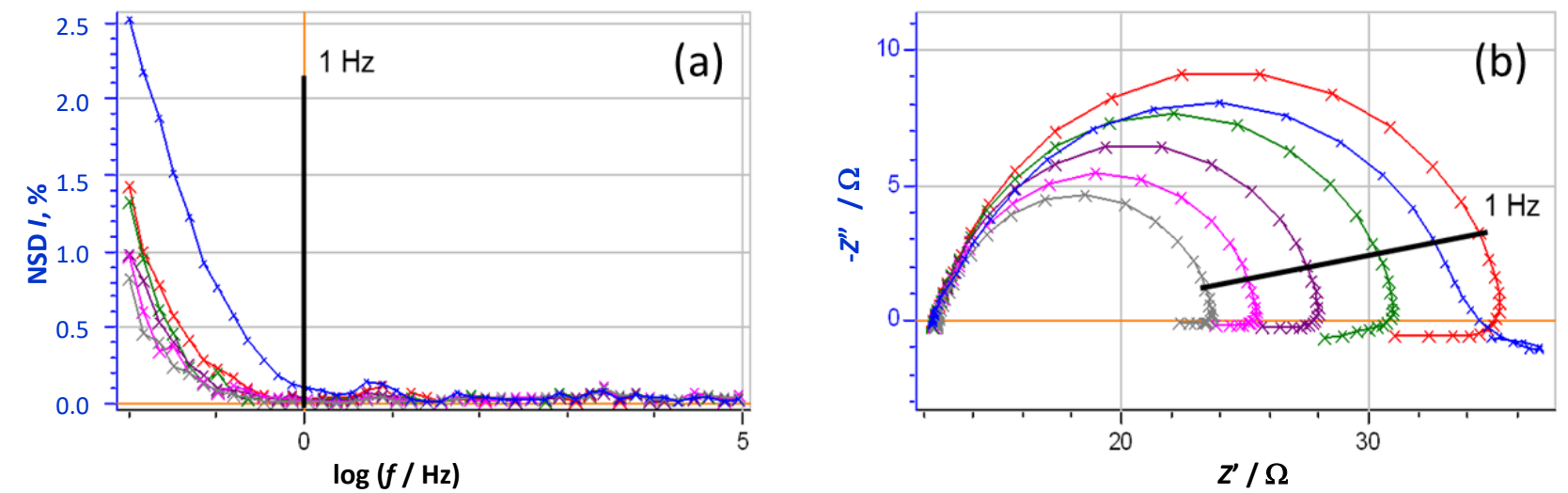

Figure 11. (a) NSD of the current response as a function of the frequency and (b) the corresponding impedance measurements. The $1 \mathrm{~Hz}$ boundary is also shown on both graphs, which could be used as a threshold value to separate stationary and quasi-non-stationary impedance data.

The NSD is dependent on the frequency. If the measurement is fast enough, it is not affected by the non-stationarity, especially by the time-variance of the system. A NSD value as low as $2.5 \%$ can have a significant effect on the shape of the impedance diagrams as it can be seen in Figure 11 . The NSD is used to determine a lower frequency limit for usable data, ie data that can be considered quasi-instantaneous, quasi-time-independent or quasi-steady-state. In Figure 11 this limit is fixed at $1 \mathrm{~Hz}$ and we consider that below this frequency the impedance measurements are affected by nonstationary phenomena.

Figure 12 shows the NSD on the potential response for the impedance data measured on a discharging battery shown in Figure 4 . It is clear that the conditions are highly non-stationary with a NSD reaching a maximum of $120 \%$ at around $100 \mathrm{mHz}$ for the first measurement (Fig. 12a) where the DC potential changes the most (Fig. 5a).

The maximum NSD is reduced drastically for the second measurement reaching $50 \%$ at $10 \mathrm{mHz}$ (Fig. 12b). The maximum NSD does not go over $4 \%$ at all frequencies from the fifth measurement (Fig. 12e). At this point, the potential does not change along the measurement (Fig. 5e), we have reached the nominal potential of the battery. At the ninth measurement, the NSD is very close to $0 \%$ for all frequencies (Fig. 12f). In the same way as for the impedance measurement on a corroding system, we can determine a lower frequency limit below which we consider that the measurements are not valid and cannot be interpreted without accounting for a time dependence. In the case of the measurement on the battery cell, this lower limit would be $10 \mathrm{~Hz}$. It is indicated as a straight line in Figure 12.

We have seen in this part that the NSD indicator could be used to indicate the non-stationarity of the system, which can lead to strong deformation of the impedance data and wrong 
interpretation. In the next part we will explain the "4D impedance" method to correct the effect of time-variance on impedance data and lead to data that can be considered as valid or quasistationary and rightly interpreted.

(a)

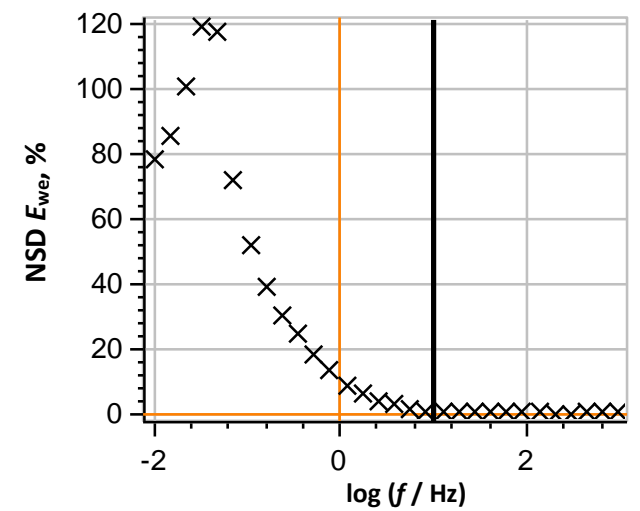

(c)

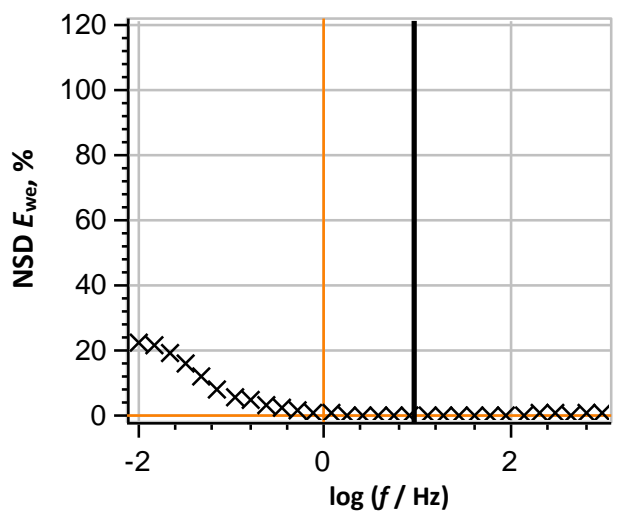

(e)

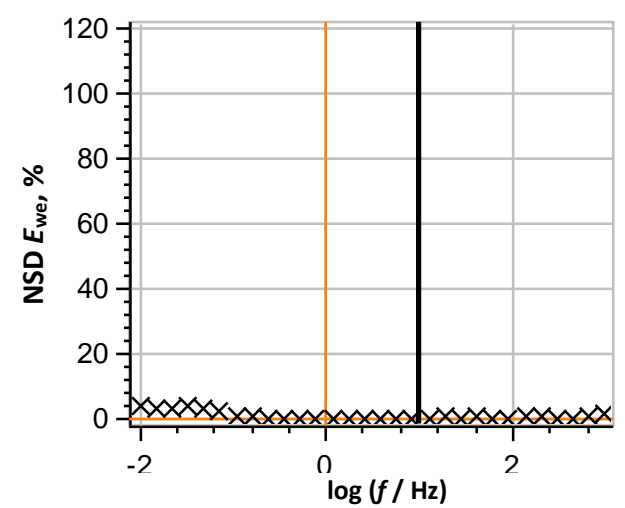

(b)

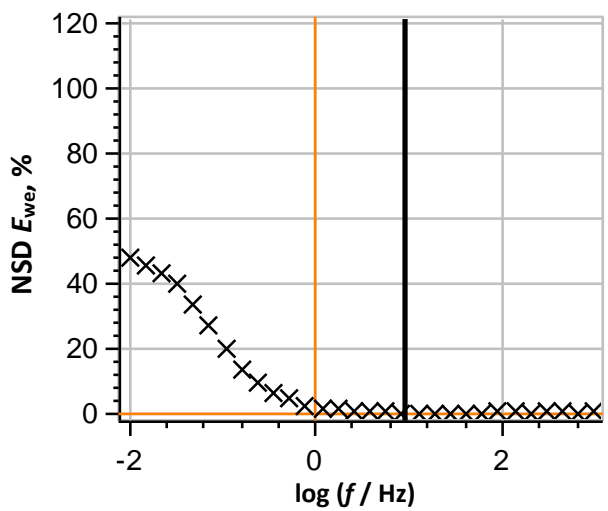

(d)

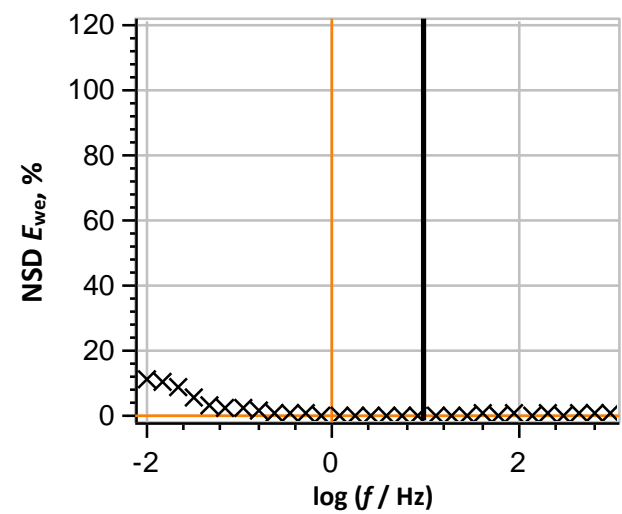

(f)

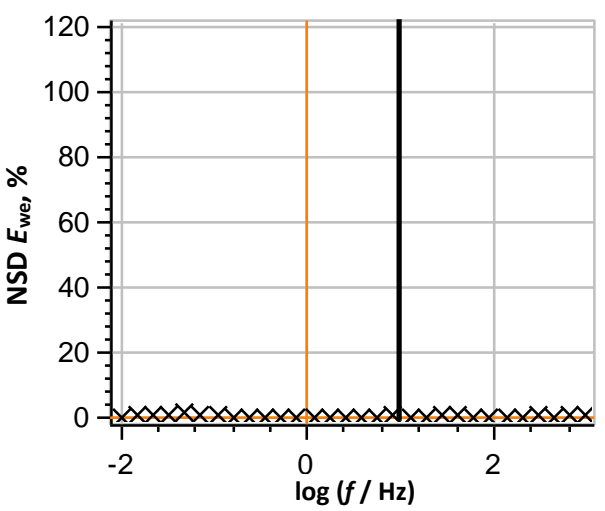

Figure 12. NSD of the potential response from impedance measurements shown in Fig. 7.

(a)-(e) Cycles 1-5 (f) Cycle 9. Even if the DC potential does not seem to change the effect of time-variance is still detectable thanks to NSD. It seems only data from $10 \mathrm{kHz}$ to $10 \mathrm{~Hz}$ can used.

\section{" $4 D$ impedance"}

This method was first introduced by Stoynov in his seminal paper [9]. Firstly, the acquired impedance data need to be represented as a function of time, as it was first shown in Fig. 2e. Figure 13a shows the results from Fig. 3a represented as a function of time.

Secondly, all data points obtained at the same frequency but at different times are interpolated to produce what we could name a temporal impedance envelope (Fig. 13b). For each frequency, $Z^{\prime}$ and $-Z^{\prime \prime}$ are expressed as a function of time. 
Thirdly, the instantaneous impedance is calculated using the interpolations obtained before. The instantaneous impedance diagram is considered as a cross-section of the temporal impedance envelope. In Figure 13c, three cross-sections are shown which are considered to be the instantaneous impedance diagrams corresponding to graphs 2, 4 and 6 in Fig. 3a, or more importantly, as impedance data of the system in the state it is at the start of frequency scans 2,4 and 6 . These data, corrected for time-variance, can now be considered valid or quasi-stationary and could be interpreted using equivalent circuit modelling for example.

This method has been recently successfully applied on various materials such as poly(oethoxyaniline) [20] and poly(3,4-ethylenedioxythiophene) [21] modified electrodes.

(a)

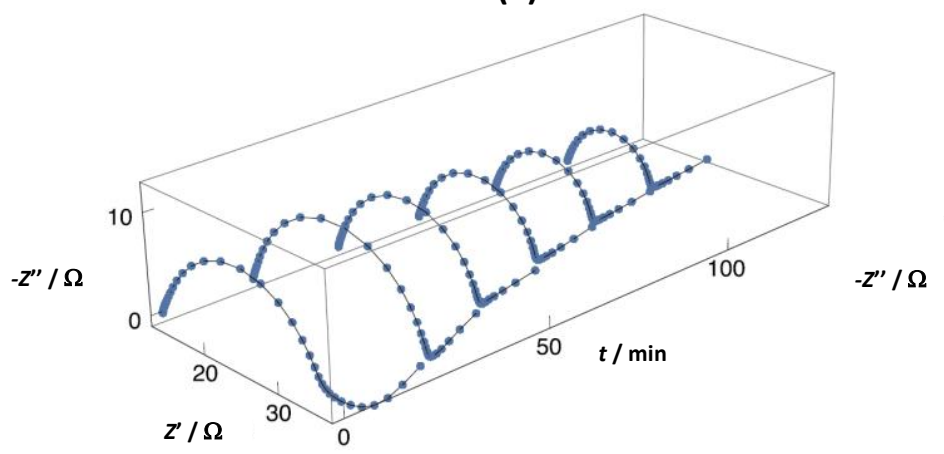

(c)

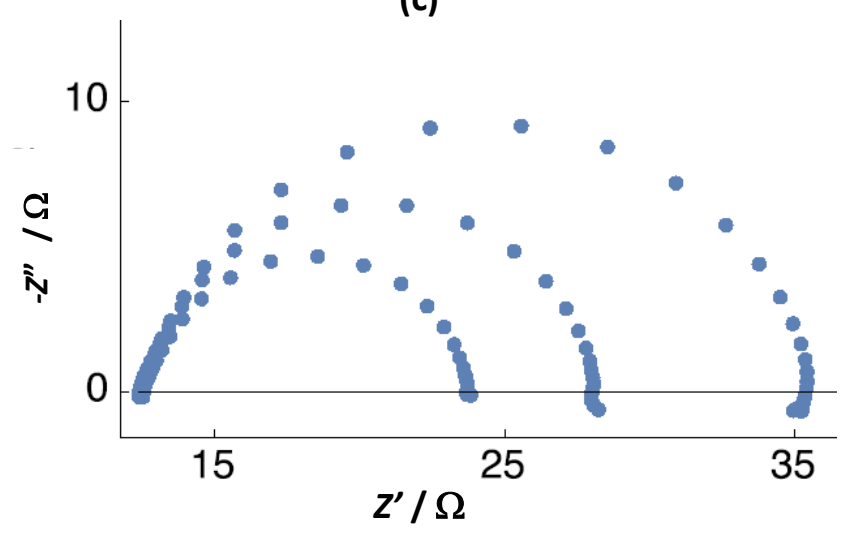

(b)

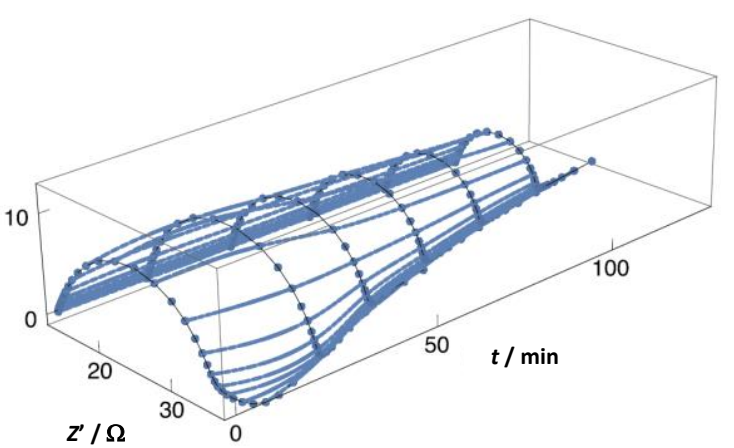

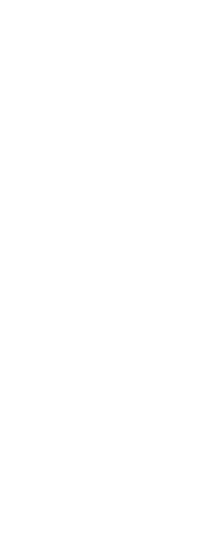

Figure 13. (a) Impedance data from Fig. 6 a plotted as a function of time. (b) Same gra phs as before but with interpolating iso-frequency curves in dots. (c) Calculated instantaneous impedance diagrams at times corresponding to the first points of graphs 2, 4 and 6 in Fig. 6 a.

\section{Conclusions}

In this paper, we have tried to show the effect of system time-variance on impedance measurements and to present some tricks and methods to both detect and correct these effects.

Using a calculation software (Mathematica [19]) we first simulated the effect of a time-changing polarization resistance on the low-frequency impedance of a simple corroding system. Experimental data obtained on a mild steel sample in sulfuric acid showed similar trends than the simulated ones.

We also showed some data obtained on a discharging battery. Impedance data can be considered stationary when the nominal voltage of the battery is reached.

Several methods available to check that impedance measurements are performed on a stationary system or not were presented: i) performing successive measurements; ii) using the NSD indicator. This indicator is also used to determine a lower frequency limit below which the data cannot be considered as valid. It is also recommended in corrosion to use galvano-controlled EIS so that the change of corrosion potential is accounted for. 
Finally, the "4D impedance" method was described in detail, which seems to be the only method to easily remove the effect of time-variance.

\section{References}

[1] M. Urquidi-Macdonald, D. Macdonald, Electrochimica Acta 35 (1990) 1559-1566.

[2] K. Darowicki, Journal of Electroanalytical Chemistry 486 (2000) 101-105.

[3] C. A. Schiller, F. Richter, E. Gülzow, N. Wagner, Physical Chemistry Chemical Physics, 3 (2001) 374378.

[4] C. Wagner and W. Traud, Zeitschrift für Elektrochemie und angewandte physikalische Physik 44 (1938) 391-402.

[5] M. Stern and A. L. Geary, Journal of the Electrochemical Society 104 (1957) 56-63.

[6] I. Epelboin, M. Keddam, H. Takenouti Journal of Applied Electrochemistry 2 (1972) 71-79.

[7] F. Mansfeld in Advances in corrosion science and technology M. G. Fontana et al. Eds., Plenum Press, New York, U.S.A., 1976, p. 163.

[8] C. Gabrielli, M. Keddam, H. Takenouti in Treatise on materials science and technology; Corrosion: aqueous processes and passive films, J. C. Scully, Ed., Academic Press Inc., London, U.-K., 1983, p. 395

[9] Z. B. Stoynov, B. S. Savova-Stoynov, T. Kossev, Journal of Power Sources 30 (1990) 275-285.

[10] F. Berthier, J.-P. Diard, A. Jussiaume, J.-J. Rameau, Corrosion Science, 30 (1990) 239-247.

[11] M. Keddam, Z. B. Stoynov, H. Takenouti, Journal of Applied Electrochemistry 7 (1977) 539-544.

[12] M. Itagaki, K. Honda, Y. Hoshi and I. Shitanda, Journal of Electroanalytical Chemistry 737 (2015) 7884.

[13] M. Itagaki, N. Kobari, S. Yotsuda, K. Watanabe, S. Kinoshita, M. Ue, Journal of Power Sources 135 (2004) 255-261.

[14] J.-P. Diard, B. Le Gorrec, C. Montella, Journal of Power Sources, 70 (1998) 78-84.

[15] C. Montella, J.-P. Diard, B. Le Gorrec, Exercices de cinétique électrochimique II. Méthode d'impédance, Hermann Eds., 2005, p. 148, p. 217.

[16] M. E. Orazem, B. Tribollet, Electrochemical Impedance Spectroscopy, Wiley \& Sons Eds., 2008, p. 165.

[17] Wolfram Research, Inc., Mathematica, Version 12.0, Champaign, IL (2019).

[18] J.-P. Diard, P. Landaud, B. Le Gorrec, C. Montella, Journal of Electroanalytical Chemistry 255 (1988) 1-20.

[19] Bio-Logic website, support section https://www.bio-logic.net/wp-content/uploads/WP2Systems.pdf (05/09/2019).

[20] V. Horvat-Radošević, K. Kvastek, K. Magdić Košiček, Bulgarian Chemical Communications 49 (2017) 119-127.

[21] D. Zalka, S. Vesztergom, M. Ujvári, G. G. Láng, Journal of Eletrochemical Science and Engineering 8 (2018) 151-162.

(C)2019 by the authors; licensee IAPC, Zagreb, Croatia. This article is an open-access article distributed under the terms and conditions of the Creative Commons Attribution license (http://creativecommons. org/licenses/by/4.0/) 